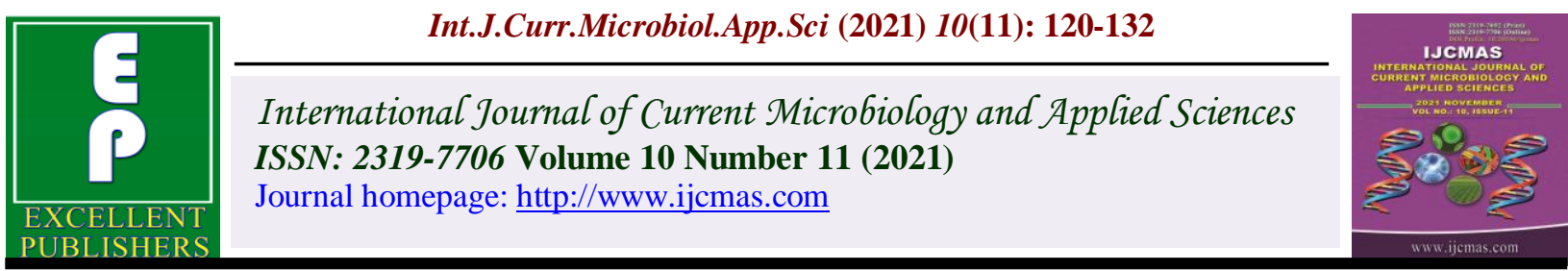

\title{
Phenotypic and Molecular Identification of Pathogenic Fusarium Species Isolated from Various Pulse Growing Geographic Areas of India
}

\author{
Monika Singh $^{1^{*}}$, O. P. Sharma ${ }^{2}$ and Someshwar Bhagat ${ }^{3}$ \\ ${ }^{1}$ Mewar University, NH-79, Gangarar, Chittorgarh, Rajasthan-312901, India \\ ${ }^{2}$ ICAR-National Research Centre for Integrated Pest Management, Lal Bahadur Shastri \\ Building, Ajantha Ave, Pusa Campus, New Delhi - 110012, India \\ ${ }^{3}$ ICAR-Central Rainfed Upland Rice Research Station (NRRI), Ranchi Patna Highway, \\ Hazaribag, Jharkhand - 825302, India \\ *Corresponding author
}

\section{A B S T R A C T}

\section{Keywords}

Fusarium sp., identification, ITSrDNA, molecular, morphology, phylogeny

\section{Article Info}

\section{Received:}

07 October 2021

Accepted:

29 October 2021

Available Online:

10 November 2021
Fusarium phythopathogenic fungi is responsible for high economic loss of cereal food crop. The objective of this study was aimed at isolation, morphological and molecular identification of Fusarium species. 13 different Fusarium spp. i.e. F. solani, F. chlamydosporum, $F$. tabacinum, $F$. fujikuroi, $F$. oxysporum, $F$. verticillioides, $F$. brachygibbosum, Fusarium sp. and $F$. incarnatum were isolated and identified from diseased samples of chickpea, pigeonpea, rice, lentil and garden pea crop. Colony characteristics like colony color, colony growth diameters, mycelium type, sporulation, pigmentation, odour were obtained after culture purification. Shape, size and septation of microconidia and macroconidia, position, shape, occurrence and size of chlamydospores, conidiophore branching were examined microscopically. MS10, BI01 and KA14 isolates were slow growing, BI02 and UP07 were moderate growing and BI03, HA04, MS06, MS09, MS11 and KA(Gul)13 were fast to very fast growing on PDA after 7-10 days. Chlamydospores were found in most of the isolates. Colonies were abundant, loosely tufted, fluffy, pannose, vinaceous floccose, powdery and some were flat appressed, arachnoid. Pigmentation of most of the isolates was pinkish white to dark pink, carmine to violet in colour. Phylogenetic analysis was done by maximum likelihood method using the ITS-rDNA region of Fusarium isolates and multiple sequence alignment of ITS DNA sequences was done using Clustal_W program and all identified sequences were submitted in NCBI GenBank database.

\section{Introduction}

Fusarium phytopathogenic fungi is one of the most extensively studied, ubiquitous and destructive phylogenetic diversified soil-borne pathogen around the world which is both saprophytic and pathogenic in nature with a vast host range including horticultural and grain crops that cause diseases like wilt, rot, and damping-off (Martyn, 2014; Zhou et al., 
2010; Xiong and Zhan, 2018 and LeBlanc et al., 2017). Members of this species complex are responsible for intense vascular wilt diseases in various plants, and also the cause of contagious diseases in humans and animals, creating a severe challenge to food security and public health (Zhang and Ma, 2017). Fusarium species are able to produce one or more mycotoxins which are secondary metabolites with varied amount of toxicity (Bottalico and Perrone, 2002) which can lead to intense immune- suppressive effect due to their multi-inhibitory properties on eukaryotic cells, including suppressing synthesis of DNA and RNA, protein, restrictive of mitochondrial function and disturbing cell division (Rocha $e t$ al., 2005). Mycotoxins are also responsible for major storage rots on food and feeds contaminating the substrates (Mohd Zainudin et al., 2008). Fusarium wilt is a major threat to production of chickpea since it is seed borne in nature (Jalali and Chand, 1991), it has high survival rate in the soil and on crop residues for up to 6 years as chlamydospores (ICRISAT, 1985) in India. Rice is the second most important cereal food crop grown and exported all over the world (Sunani et al., 2019) and India ranks second in rice production with an area of around 44.6 million hectare (mha) and productivity of $2972 \mathrm{~kg} / \mathrm{ha}$ (FAO, 2014). Bakanae disease caused by Fusarium fujikuroi Nirenberg severely affects quality and production in Basmati rice (Bashyal et al., 2016a, b; Gupta et al., 2015).

The most preferred method is morphological identification of Fusarium spp. is microscopic examination under light microscopy where diagnosis depend only upon morphology based on their micro and macroscopic features of diseased tissues. However, these features are mostly reported to be unstable (Booth, 1971).Usually, disease diagnosis is performed first after the appearance of visual symptoms. In disease diagnosis, biochemical and allozyme characteristics etc. are also performed which require high expertise and are still prone to inaccuracy (Kheterpal, 2006). These methods are also time consuming and proved to be limited and insufficient. At present, identification of pathogens is executed based on the nucleotide sequence information from conserved regions using PCR amplification assays, internal transcribed spacer (ITS) region from the conserved ribosomal RNA genes, translation elongation factor (EF-1a), intergenic spacer (IGS), btubulin region and the small subunit mitochondrial sequences (mtSSU) have been proved valuable in distinguishing species and origins of Fusarium (Baayen et al., 2000; O'Donnell et al., 2000; Skoygaard et al., 2001). This sequence information has been extensively used in the taxonomy and phylogenetic study of Fusarium species by identifying and discriminating isolates within a species. These molecular techniques based on DNA have been extensively used and has proved to be more rapid, sensitive and precise detection method (Sunani et al., 2019).

Therefore, the objectives of this present study were to isolate and identify disease causing Fusarium species from infected parts of crop plants based on the morphological data and molecularly using internal transcribed spacer (ITS).

\section{Materials and Methods}

The infected stems, seeds and roots were congregate from chickpea, pigeon pea, rice, lentil and garden pea fields. They were collected from different states of India i.e. Bihar, Maharashtra, Karnataka, Uttar Pradesh, Haryana and Himachal Pradesh.

\section{Isolation of Fusarium spp.}

5 to $10 \mathrm{~cm}$ segments of infected stems were collected and brought to the laboratory for isolation. Diseased stems, seeds and roots 
were aseptically cut into $1-\mathrm{cm}$ pieces and were surface sterilized in $10 \%(\mathrm{w} / \mathrm{v})$ sodium hypochlorite solution for $1 \mathrm{~min}$ each. They were rinsed in sterile distilled water and blotted dry in sterilized blotting paper then placed onto potato dextrose agar (Himedia) plates by tissue segment method (Rangaswami, 1958). PDA media was amended with streptomycin sulfate to remove bacterial contamination. Plates then were at $25^{\circ} \mathrm{C}$ in the dark. The Fusarium cultures were purified by repeated subculturing on PDA (Leslie and Summerell, 2006) and pure cultures were maintained at $4^{\circ} \mathrm{C}$ in PDA slants.

\section{Morphological identification of Fusarium isolates}

Morphological identifications of isolates were made using the criteria of Gerlach and Nirenberg (1982) and Leslie and Summerell (2006). Culture characteristics were determined by using Methuen handbook of colour chart (Kornerup and Wancher, 1978). Shape, size and septation of microconidia; shape, presence or absence of microconidia and presence or absence of chlamydospores based on Summeral et al., (2003) were taken.

\section{Molecular identification of Fusarium isolates}

\section{Isolation of DNA}

Genomic DNA was extracted by method described by Raeder and Broda (1985). For DNA extraction, 4 days old culture of Fusarium isolates were scraped from PDA plates and transferred into centrifuge tubes carrying $25 \mathrm{ml}$ pre heated $\left(65^{\circ} \mathrm{C}\right)$ lysis buffer. The tubes were incubated at $65^{\circ} \mathrm{C}$ for an hour with occasional stirring by vortexing every 15 min followed by addition of equal volume of Phenol: Chloroform: Iso-amyl alcohol (25:24:1). Samples were centrifuged at 15,000 rpm for 10 minutes at room temperature. Upper aqueous phase was precipitated with 2 volumes of ice-cold ethanol and 0.1 volume of $3 \mathrm{M}$ sodium acetate ( $\mathrm{pH} 5.20)$ and incubate for $1 \mathrm{hr}$ at $4^{\circ} \mathrm{C}$ and spinned at $15,000 \mathrm{rpm}$ for 15 minutes at room temperature and the pellets obtained were washed with $70 \%$ ethanol and again spinned at $10,000 \mathrm{rpm}$ for 10 minutes. Nucleic acid was eluted by elution buffer $(10 \mathrm{mM}$ TrisHCl and $1 \mathrm{mM}$ sodium EDTA, $\mathrm{pH}$ 8). The DNA was dissolved and it was treated with $10 \mu \mathrm{l}$ RNase and incubated for $1 \mathrm{hr}$ at $37^{\circ} \mathrm{C}$, after incubation $500 \mu \mathrm{l}$ of water saturated phenol: chloroform: isoamyl alcohol (25: 24: 1) was added and centrifuged at $10,000 \mathrm{rpm}$ for $20 \mathrm{~min}$.

The upper aqueous phase was collected and 50 $\mu \mathrm{l}$ of $3 \mathrm{M}$ sodium acetate and $1 \mathrm{ml}$ ethanol was added. Isolated DNA was incubated overnight or at $-80^{\circ} \mathrm{C}$ for $1 \mathrm{hr}$. Supernatant was discarded and the pellet is washed with $70 \%$ ethanol, air dried and approx. $100 \mu 1$ of $1 \mathrm{X}$ TE buffer was added to dissolve the pellet. DNA was then stored at $-20^{\circ} \mathrm{C}$ for PCR.

\section{Molecular characterization using ITS}

Molecular identification of Fusarium cultures were carried out by amplification of internal transcribed spacer 1, partial sequence, 5.8S ribosomal RNA gene and internal transcribed spacer 2, complete sequence and $28 \mathrm{~S}$ ribosomal RNA gene, partial sequence using primer pair ITS1 (5' TCCGTAGGTGA ACCTGCGG-3') and ITS4 (5'-TCCTCCG CTTATTGATATGC-3') (White et al., 1990).

The ITS region was amplified with an initial denaturation for 5 minutes at $95^{\circ} \mathrm{C}$, followed by 35 cycles of denaturation at $94^{\circ} \mathrm{C}$ for 1 minute, annealing of primers at $60^{\circ} \mathrm{C}$ for 30 seconds, and extension at $72^{\circ} \mathrm{C}$ for 30 seconds, and the amplification was completed with one cycle of final extension at $72^{\circ} \mathrm{C}$ for 5 minutes in PCR thermocycler (Bioer) using 25 
$\mu 1$ PCR mastermix containing 60-100 ng genomic DNA, $2.5 \mu \mathrm{l}$ of $10 \mathrm{X}$ buffer (10 mMTris-HCl, $\mathrm{pH} 8.8), 2 \mathrm{mM}$ each of dNTP, $2.5 \mathrm{mM} \mathrm{MgCl} 2,25 \mathrm{pmol} \mathrm{ml}^{-1}$ primer (each primer) and $1 \mathrm{U}$ of Taq DNA Polymerase. Amplified products were visualized on agarose gel (1.5\%) in 1X TAE buffer. The gel image was taken using gel documentation system.

\section{Sequencing and Phylogenetic analysis}

Unpurified PCR product was sent to Xcelris Labs, Gujarat, India for sequencing. The partial ITS sequence thus obtained was analyzed using BLASTn (Basic Local Alignment Search Tool for Nucleotide Sequences) with NCBI (National Center for Biotechnology information; www.ncbi.nih.gov), GenBank database to assess its identity. Alignment of ITS DNA sequences was done using Clustal_W program (Vincelli and Tisserat, 2008). The evolutionary analysis was conducted in MEGA X (Kumar et al., 2018) and was inferred by using the Maximum Likelihood method and Tamura-Nei model (Tamura and Nei, 1993). This analysis involved 13 nucleotide sequences. There were a total of 524 positions in the final dataset.

\section{Results and Discussion}

\section{Isolation and morphological identification} of Fusarium isolates

13 Fusarium spp. were isolated from diseased crop plants. The details of location, host of Fusarium isolates are shown in table 1.

Based on colony characteristics and microscopic structures of microconidia, macroconidia and other morphological characters, BI01 was identified as $F$. solani, $\mathrm{BI02}$ as $F$. chlamydosporum, $\mathrm{BI03}$ as $F$. fujikuroi, HA04 as $F$. fujikuroi, MS05 as $F$. tabacinum, MS06 as $F$. solani, UP07 as $F$. oxysporum, MS09 as $F$. verticillioides, MS10 as $F$. brachygibbosum, MS11 as $F$. solani, $\mathrm{KA}(\mathrm{Gul}) 13$ as Fusarium sp., KA14 as $F$. solani and $\mathrm{HP}(\mathrm{Ham}) 15$ as $F$. incarnatum(Table 2).

\section{Molecular identification based on ITS}

PCR amplification of ITS region generated bands ranging from 460-504 bp Fusarium sequences thus obtained were compared with sequences from NCBI database using BLASTn.

These sequences thus identified were deposited in NCBI GenBank and accession numbers were allotted. Table 1 shows isolate code with GenBank accession numbers.

Isolate BI01, MS06, MS11 and KA14 were identified as $F$. solani (KP881505, KP881510, KP881514 and KP881516 respectively), BI02 as $F$. chlamydosporum (KP881506), BI03 and HA04 as $F$. fujikuroi (KP881507 and KP881508), MS05 as $F$. proliferatum (KP881509), UP07 as $F$. oxysporum (KP881511), MS09 as F.verticillioides (KP881512), MS10 as F. brachygibbosum (KP881513) and KA(Gul)13 and HP(Ham)15 as Fusarium sp. (KP881515 and KP881517).

The evolutionary history of Fusarium isolates was inferred by using the Maximum Likelihood method and Tamura-Nei model (Tamura and Nei, 1993).

The tree is drawn to scale, with branch lengths measured in the number of substitutions per site. This analysis involved 13 nucleotide sequences. Codon positions included were $1 \mathrm{st}+2 \mathrm{nd}+3 \mathrm{rd}+$ Noncoding. There were a total of 524 nucleotide sites in the final dataset. Evolutionary analyses were conducted in MEGA X (Kumar et al., 2018). 
Table.1 Location, host, morphological and sequenced based identified species with GenBank accessions of Fusarium isolates

\begin{tabular}{|c|c|c|c|c|c|}
\hline Isolate code & Location & Host plant & $\begin{array}{c}\text { Morphological } \\
\text { identification }\end{array}$ & $\begin{array}{l}\text { Sequence based } \\
\text { identification }\end{array}$ & $\begin{array}{c}\text { GenBank } \\
\text { Accession No. }\end{array}$ \\
\hline BI01 & Samastipur, Bihar & Lentil stem & Fusarium solani & Fusarium solani & KP881505 \\
\hline BI02 & Samastipur, Bihar & Lentil stem & $\begin{array}{c}\text { Fusarium } \\
\text { chlamydosporum }\end{array}$ & $\begin{array}{c}\text { Fusarium } \\
\text { chlamydosporum }\end{array}$ & KP881506 \\
\hline BI03 & Samastipur, Bihar & Lentil stem & $\begin{array}{l}\text { Fusarium } \\
\text { fujikuroi }\end{array}$ & Fusarium fujikuroi & KP881507 \\
\hline HA04 & Kaithal, Haryana & Rice stem & Fusariumfujikuroi & Fusariumfujikuroi & KP881508 \\
\hline MS05 & $\begin{array}{l}\text { Badnapur, } \\
\text { Maharashtra }\end{array}$ & $\begin{array}{l}\text { Pigeon pea } \\
\text { stem }\end{array}$ & $\begin{array}{l}\text { Fusarium } \\
\text { tabacinum }\end{array}$ & Fusariumproliferatum & KP881509 \\
\hline MS06 & Bori, Maharashtra & $\begin{array}{c}\text { Chickpea } \\
\text { stem }\end{array}$ & Fusarium solani & Fusarium solani & KP881510 \\
\hline UP07 & Kanpur (U.P.) & $\begin{array}{l}\text { Chickpea } \\
\text { seed }\end{array}$ & $\begin{array}{l}\text { Fusarium } \\
\text { oxysporum }\end{array}$ & Fusarium oxysporum & KP881511 \\
\hline MS09 & $\begin{array}{l}\text { Badnapur, } \\
\text { Maharashtra }\end{array}$ & $\begin{array}{l}\text { Chickpea } \\
\text { stem }\end{array}$ & $\begin{array}{c}\text { Fusarium } \\
\text { verticillioides }\end{array}$ & $\begin{array}{c}\text { Fusarium } \\
\text { verticillioides }\end{array}$ & KP881512 \\
\hline MS10 & Jalna, Maharashtra & $\begin{array}{l}\text { Pigeon pea } \\
\text { stem }\end{array}$ & $\begin{array}{c}\text { Fusarium } \\
\text { brachygibbosum }\end{array}$ & $\begin{array}{c}\text { Fusarium } \\
\text { brachygibbosum }\end{array}$ & KP881513 \\
\hline MS11 & Jalna, Maharashtra & $\begin{array}{l}\text { Pigeon pea } \\
\text { stem }\end{array}$ & Fusarium solani & Fusarium solani & KP881514 \\
\hline KA(Gul)13 & $\begin{array}{l}\text { Gulbarga, } \\
\text { Karnataka }\end{array}$ & $\begin{array}{l}\text { Pigeon pea } \\
\text { stem }\end{array}$ & Fusarium sp. & Fusarium sp. & KP881515 \\
\hline KA14 & Raichur, Karnataka & $\begin{array}{l}\text { Pigeon pea } \\
\text { root }\end{array}$ & Fusarium solani & Fusarium solani & KP881516 \\
\hline HP(Ham)15 & Hamirpur (H.P.) & $\begin{array}{c}\text { Garden pea } \\
\text { stem }\end{array}$ & $\begin{array}{l}\text { Fusarium } \\
\text { incarnatum }\end{array}$ & Fusarium sp. & KP881517 \\
\hline
\end{tabular}

Fig.1 Phylogenetic tree generated by Maximum Likelihood method using the ITS region nucleotide sequence of the conserved ribosomal DNA of Fusarium isolates.

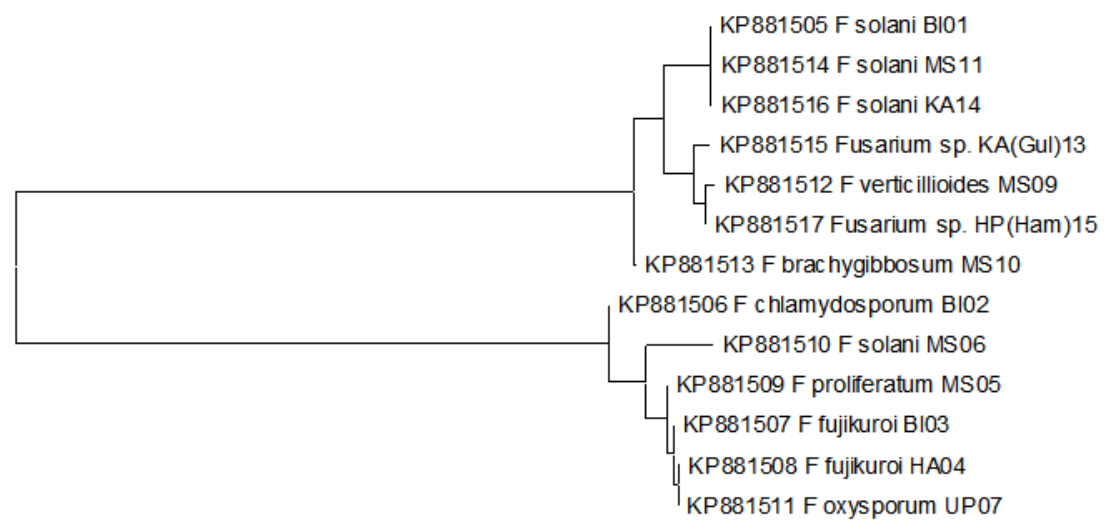


Table.2 Anamorphic characteristics of Fusarium isolates

\begin{tabular}{|c|c|c|c|c|c|c|c|}
\hline $\begin{array}{l}\text { Isolate } \\
\text { code }\end{array}$ & $\begin{array}{c}\text { Colonieson } \\
\text { PDA }\end{array}$ & $\begin{array}{c}\text { Mycelium } \\
\text { type }\end{array}$ & $\begin{array}{l}\text { Pigmentati } \\
\text { on }\end{array}$ & Sporulation & Conidiophore & Conidia & Chlamydospore \\
\hline BI01 & $\begin{array}{l}\text { slow growing, } \\
\text { reaching } 4.0- \\
4.2 \mathrm{~cm} \text { diam. } \\
\text { in } 10 \text { days }\end{array}$ & $\begin{array}{l}\text { floccose or } \\
\text { tufted, } \\
\text { cottony }\end{array}$ & $\begin{array}{l}\text { whitish to } \\
\text { light pink }\end{array}$ & Less & $\begin{array}{l}\text { Formed in the aerial } \\
\text { mycelium, first } \\
\text { unbranched, later loosely } \\
\text { long branched sometimes } \\
\text { densely aggregated }\end{array}$ & $\begin{array}{l}\text { almost straight to } \\
\text { slightly curved, thick } \\
\text { walled, widest at } \\
\text { centre, cuneiform, } 0-2 \\
\text { septate }(3.5-5.2 \mu \mathrm{m})\end{array}$ & $\begin{array}{l}\text { round walled, } \\
\text { globose to } \\
\text { subglobose, } \\
\text { single }(5-12 \mu \mathrm{m}), \\
\text { terminal }\end{array}$ \\
\hline BI02 & $\begin{array}{l}\text { moderate } \\
\text { growing, } \\
\text { reaching 5-5.2 } \\
\text { cm diam. in } 7 \\
\text { days }\end{array}$ & $\begin{array}{l}\text { profuse, } \\
\text { densely } \\
\text { floccose, } \\
\text { powdery at } \\
\text { surface }\end{array}$ & $\begin{array}{l}\text { pink, } \\
\text { carmine }\end{array}$ & $\begin{array}{l}\text { microconidia formed } \\
\text { after } 4 \text { days }\end{array}$ & $\begin{array}{l}\text { primary conidiophore } \\
\text { arising at right angle } \\
\text { from main conidiophore }\end{array}$ & $\begin{array}{l}\text { microconidia ovoid, } 1- \\
3 \text { septate }(12-15 \times 3.0- \\
3.2 \mu \mathrm{m}), \text { widest at } \\
\text { centre }\end{array}$ & $\begin{array}{l}\text { Many, globose } \\
\text { to subglobse, } \\
\text { terminal or } \\
\text { intercalary seen, } \\
\text { single }(7-17 \mu \mathrm{m}) \\
\text { or in pair and in } \\
\text { chain }\end{array}$ \\
\hline BI03 & $\begin{array}{l}\text { fast growing, } \\
\text { reaching } 7.5-8 \\
\text { cm diam. in } 7 \\
\text { days }\end{array}$ & $\begin{array}{l}\text { loosely } \\
\text { pannose, } \\
\text { vinaceous }\end{array}$ & dark violet & $\begin{array}{c}\text { microconidia start } \\
\text { forming quickly and } \\
\text { later } \\
\text { macroconidiaformed }\end{array}$ & $\begin{array}{c}\text { primary conidiophores } \\
\text { arising laterally from } \\
\text { hyphae in the aerial } \\
\text { mycelium }\end{array}$ & $\begin{array}{c}1 \text { or } 2 \text { septate }(4-13 \mathrm{x} \\
1.5-4.5 \mu \mathrm{m}) \\
\text { microconidia, } \\
\text { macroconidia slender, } \\
\text { falcate or straight }\end{array}$ & Absent \\
\hline HА04 & $\begin{array}{l}\text { very fast } \\
\text { growing, } \\
\text { reaching } 8-9 \\
\text { cm diam. in } 7 \\
\text { days }\end{array}$ & $\begin{array}{c}\text { pale } \\
\text { vinaceous }\end{array}$ & $\begin{array}{l}\text { dark pink to } \\
\text { violet }\end{array}$ & $\begin{array}{c}\text { abundant } \\
\text { macroconidiaand } \\
\text { microconidia formed }\end{array}$ & $\begin{array}{l}\text { primary conidiophores } \\
\text { arising from hyphae, } \\
\text { secondary conidiophores } \\
\text { are densely branched }\end{array}$ & $\begin{array}{c}\text { straight microconidia } 1 \\
\text { or } 2 \text { septate }(4-13 \mathrm{x} \\
1.5-4.5 \mu \mathrm{m}), \text { sickle } \\
\text { shaped macroconidia, } \\
3-5 \text { septate }(19-52 \times \\
2.0-4.2 \mu \mathrm{m}),\end{array}$ & Absent \\
\hline MS05 & $\begin{array}{l}\text { moderately fast } \\
\text { growing, } \\
\text { reaching } 7-8 \\
\text { cm diam. in } 7 \\
\text { days }\end{array}$ & $\begin{array}{l}\text { fluffy, } \\
\text { arachnoid, } \\
\text { floccose }\end{array}$ & $\begin{array}{l}\text { pinkish } \\
\text { white }\end{array}$ & abundant & $\begin{array}{l}\text { at first arising in the } \\
\text { aerial mycelium as lateral } \\
\text { phialides, sparsely } \\
\text { branched }\end{array}$ & $\begin{array}{c}\text { mostly single septate } \\
(5-16 \text { x } 2.1-4.1 \mu \mathrm{m}), \\
\text { slightly curved, with } \\
\text { pointed apical cell, } 1 \\
\text { celled with } 2 \text { vacuoles, } \\
\text { variable shape }\end{array}$ & Not formed \\
\hline
\end{tabular}




\begin{tabular}{|c|c|c|c|c|c|c|c|}
\hline MS06 & $\begin{array}{l}\text { fast growing, } \\
\text { reaching } 7.8- \\
8.2 \mathrm{~cm} \text { diam. } \\
\text { in } 10 \text { days }\end{array}$ & $\begin{array}{l}\text { floccose, } \\
\text { sparse, } \\
\text { cottony, } \\
\text { creamish } \\
\text { white }\end{array}$ & Dull white & $\begin{array}{l}\text { Abundant } \\
\text { macroconidia }\end{array}$ & $\begin{array}{l}\text { primary conidiophores } \\
\text { unbranched, secondary } \\
\text { conidiophore densely }\end{array}$ & $\begin{array}{c}\text { falvatemacroconidia, } 3- \\
5 \text { septate }(20-38 \times 3.3- \\
6.0 \mu \mathrm{m}) \\
\text { microconidiaoval, } \\
\text { ellipsoid, reniform, } 0-1 \\
\text { septate }(6-10 \times 2.7- \\
3.3 \mu \mathrm{m})\end{array}$ & $\begin{array}{l}\text { Mostly seen, } \\
\text { terminal or } \\
\text { intercalary, } \\
\text { globose to } \\
\text { subglobose }\end{array}$ \\
\hline UP07 & $\begin{array}{l}\text { moderate } \\
\text { growing, } \\
\text { reaching } 6.5- \\
7.0 \mathrm{~cm} \text { diam. } \\
\text { in } 8 \text { days }\end{array}$ & $\begin{array}{l}\text { dense, } \\
\text { arachnoid, } \\
\text { pink peach } \\
\text { with violet } \\
\text { tinge }\end{array}$ & $\begin{array}{l}\text { rose pink to } \\
\text { strong } \\
\text { purple }\end{array}$ & $\begin{array}{l}\text { microconidia } \\
\text { emerging from aerial } \\
\text { mycelium in false } \\
\text { heads, macroconidia } \\
\text { solitarily scattered }\end{array}$ & $\begin{array}{l}\text { primary conidiophores } \\
\text { short, secondary } \\
\text { conidiophore dense, } \\
\text { verticillately branched }\end{array}$ & $\begin{array}{c}\text { microcondia } 1 \text { or } 2 \\
\text { septate }(8-22 \times 2.3-4.5 \\
\mu \mathrm{m}), \text { ellipsoid, oval, } \\
\text { slightly curved, } \\
\text { macroconidia falcate, } \\
\text { subcylindric, mostly } \\
\text { 3septate }(18-54 \times 2.7- \\
6.0 \mu \mathrm{m}),\end{array}$ & $\begin{array}{c}\text { abundant, in } \\
\text { hyphae and } \\
\text { conidia, terminal } \\
\text { seen, rough } \\
\text { walled, globose } \\
\text { to subglobose, } \\
\text { single. }\end{array}$ \\
\hline MS09 & $\begin{array}{l}\text { very fast } \\
\text { growing, } \\
\text { reaching } 7.8- \\
8.2 \mathrm{~cm} \text { diam. } \\
\text { in } 8 \text { days }\end{array}$ & $\begin{array}{l}\text { abundant, } \\
\text { loosely } \\
\text { tufted, } \\
\text { white } \\
\text { pinkish } \\
\text { later } \\
\text { vinaceous }\end{array}$ & $\begin{array}{l}\text { dark } \\
\text { vinaceous, } \\
\text { pink to } \\
\text { purple }\end{array}$ & $\begin{array}{l}\text { starts quickly, } \\
\text { microconidia in long } \\
\text { chains, bearing } \\
\text { macroconidia lately }\end{array}$ & $\begin{array}{l}\text { primary conidiophores } \\
\text { arising from hyphae of } \\
\text { aerial mycelium, } \\
\text { secondary conidiophores } \\
\text { densely branched }\end{array}$ & $\begin{array}{c}\text { microconidia sharp } \\
\text { pointed at corners, } 1 \text { or } \\
2 \text { septate }(4-19 \times 1.5- \\
4.6 \mu \mathrm{m}) \text {, macroconidia, } \\
\text { broadest just below the } \\
\text { apical cell, constricted, } \\
3 \text { septate }(18-56 \times 2.1- \\
4.0 \mu \mathrm{m})\end{array}$ & Absent \\
\hline MS10 & $\begin{array}{l}\text { slow growing, } \\
\text { reaching } 5.4- \\
6.5 \mathrm{~cm} \text { diam. } \\
\text { in } 8 \text { days }\end{array}$ & $\begin{array}{l}\text { powdery, } \\
\text { white and } \\
\text { rose, } \\
\text { concentric } \\
\text { ring, violet } \\
\text { at centre }\end{array}$ & pink to dark & late conidia formed & $\begin{array}{l}\text { microconidia lacking in } \\
\text { aerial mycelium }\end{array}$ & $\begin{array}{c}\text { microconidia } 1-2 \\
\text { septate mostly }(1.8-3.6 \\
\text { x } 11.1-15.8 \mu \mathrm{m}), \text { ovoid } \\
\text { to fusiform, septate } \\
\text { spores slightly curved, } \\
\text { scattered macroconidia } \\
\text { not very abundant, } 2 \\
\text { septate seen, }\end{array}$ & $\begin{array}{l}\text { terminal or } \\
\text { intercalary, } \\
\text { rough walled, } \\
\text { single seen, } \\
\text { globose, } \\
\text { generally 1- } \\
\text { celled (10-15.1 } \\
\mu \mathrm{m}), \text { granular }\end{array}$ \\
\hline MS11 & $\begin{array}{l}\text { fast growing, } \\
\text { reaching } 7.8-\end{array}$ & $\begin{array}{l}\text { floccose, } \\
\text { cottony, felt }\end{array}$ & $\begin{array}{l}\text { pink, later } \\
\text { red-brown }\end{array}$ & $\begin{array}{l}\text { starts very quickly in } \\
\text { aerial mycelium, }\end{array}$ & $\begin{array}{l}\text { primary conidiophores } \\
\text { arising laterally from }\end{array}$ & $\begin{array}{l}\text { microconidia oval, } \\
\text { ellipsoid, } 0-1 \text { septate }\end{array}$ & $\begin{array}{l}\text { globose to } \\
\text { subglobose, }\end{array}$ \\
\hline
\end{tabular}




\begin{tabular}{|c|c|c|c|c|c|c|c|}
\hline & $\begin{array}{l}8.2 \mathrm{~cm} \text { diam. } \\
\text { in } 8 \text { days }\end{array}$ & $\begin{array}{l}\text { like, white } \\
\text { to pinkish }\end{array}$ & & $\begin{array}{l}\text { microconidia } \\
\text { cohering in false } \\
\text { heads, macroconidia } \\
\text { formed profusely }\end{array}$ & $\begin{array}{l}\text { hyphae of aerial } \\
\text { mycelium, secondary } \\
\text { conidiophores densely, } \\
\text { irregularly branched }\end{array}$ & $\begin{array}{l}(\mu \mathrm{m}), \text { macroconidia } \\
\text { straight cylindrical } \\
\text { central part, with } \\
\text { elongated, hooked, } \\
\text { pointed corners } 3-5 \\
\text { septate }(\mu \mathrm{m})\end{array}$ & $\begin{array}{l}\text { single }(6-10 \mu \mathrm{m}) \\
\text { seen, smooth } \\
\text { walled, attached } \\
\text { to hyphae }\end{array}$ \\
\hline $\begin{array}{c}\text { KA(Gul }) 1 \\
\mathbf{3}\end{array}$ & $\begin{array}{l}\text { fast growing, } \\
\text { reaching } 7.5- \\
8.0 \mathrm{~cm} \text { diam. } \\
\text { in } 10 \text { days }\end{array}$ & $\begin{array}{l}\text { densely } \\
\text { floccose to } \\
\text { funiculose, } \\
\text { arachnoid }\end{array}$ & $\begin{array}{l}\text { pinkish, } \\
\text { become rosy } \\
\text { to purple }\end{array}$ & $\begin{array}{l}\text { starts quickly, } \\
\text { confluent growth }\end{array}$ & $\begin{array}{l}\text { initially arising as single } \\
\text { lateral phialide, later in } \\
\text { pionnates densely } \\
\text { verticillately branched, }\end{array}$ & $\begin{array}{l}\text { thin-walled ovoid, } \\
\text { fusoid, } 1 \text { or } 2 \text { celled } \\
\text { conidia, taller } \\
\text { macroconidia } 3 \text { septate } \\
(17-42 \times 2.0-3.9 \mu \mathrm{m}),\end{array}$ & $\begin{array}{c}\text { globose to } \\
\text { subglobose, } \\
\text { smooth walled, } \\
\text { single }(8-12 \mu \mathrm{m}) \\
\text { or in clusters }\end{array}$ \\
\hline KA14 & $\begin{array}{l}\text { slow growing, } \\
\text { reaching } 5.5- \\
6.0 \mathrm{~cm} \text { diam. } \\
\text { in } 7 \text { days }\end{array}$ & $\begin{array}{l}\text { floccose, } \\
\text { felt-like, } \\
\text { pink to light } \\
\text { greyish } \\
\text { colony }\end{array}$ & $\begin{array}{l}\text { red- brown, } \\
\text { greyish }\end{array}$ & $\begin{array}{l}\text { starts quickly in } \\
\text { aerial mycelium, } \\
\text { abundant } \\
\text { macroconidia early } \\
\text { formed }\end{array}$ & $\begin{array}{l}\text { primary conidiophores } \\
\text { arising laterally from } \\
\text { hyphae of aerial } \\
\text { mycelium, secondary } \\
\text { conidiophores densely, } \\
\text { irregularly }\end{array}$ & $\begin{array}{l}\text { microconidia oblong, } \\
\text { oval, single or no } \\
\text { septate }(8-30 \times 3.0-4.2 \\
\mu \mathrm{m}), \text { subcylindric, } \\
\text { macroconidia falcate, } \\
2-3 \text { septate }(20-43 \mathrm{x} \\
3.3-6.0 \mu \mathrm{m}), \text { pointed } \\
\text { apical cell or curved at } \\
\text { corners }\end{array}$ & $\begin{array}{l}\text { terminal seen, } \\
\text { rough-walled, } \\
\text { globose to } \\
\text { subglobose, } \\
\text { single }(5-9 \mu \mathrm{m}) \text {. }\end{array}$ \\
\hline $\begin{array}{c}\text { HP(Ham) } \\
15\end{array}$ & $\begin{array}{l}\text { fast growing, } \\
\text { reaching } 7.5- \\
8.0 \mathrm{~cm} \text { diam. } \\
\text { in } 10 \text { days }\end{array}$ & $\begin{array}{l}\text { abundant, } \\
\text { densely } \\
\text { floccose, } \\
\text { powdery, } \\
\text { pinkish } \\
\text { white } \\
\text { colony }\end{array}$ & $\begin{array}{l}\text { whitish, } \\
\text { peach }\end{array}$ & $\begin{array}{l}\text { scattered only in } \\
\text { aerial mycelium }\end{array}$ & $\begin{array}{l}\text { loosely, irregularly } \\
\text { branched, } \\
\text { phialidesmonophialidic }\end{array}$ & $\begin{array}{l}\text { thick walled, hook } \\
\text { shaped apical cell at } \\
\text { corners, mostly } 3 \\
\text { septate }(13-43 \times 2.5-6.5 \\
\mu \mathrm{m}) \text { macroconidia and } \\
2 \text { septate }(8-22 \times 2.0- \\
4.5 \mu \mathrm{m}) \text { microconidia }\end{array}$ & $\begin{array}{l}\text { terminal seen, } \\
\text { rough-walled, } \\
\text { single }(5-8 \mu \mathrm{m}), \\
\text { globose to } \\
\text { subglobose }\end{array}$ \\
\hline
\end{tabular}


Fig.2 Multiple sequence alignment of 13 different Fusarium sp. isolates using conserved ribosomal ITS region. Insertion/deletion are indicated by dashes (-) and identical nucleotide by dots $($.

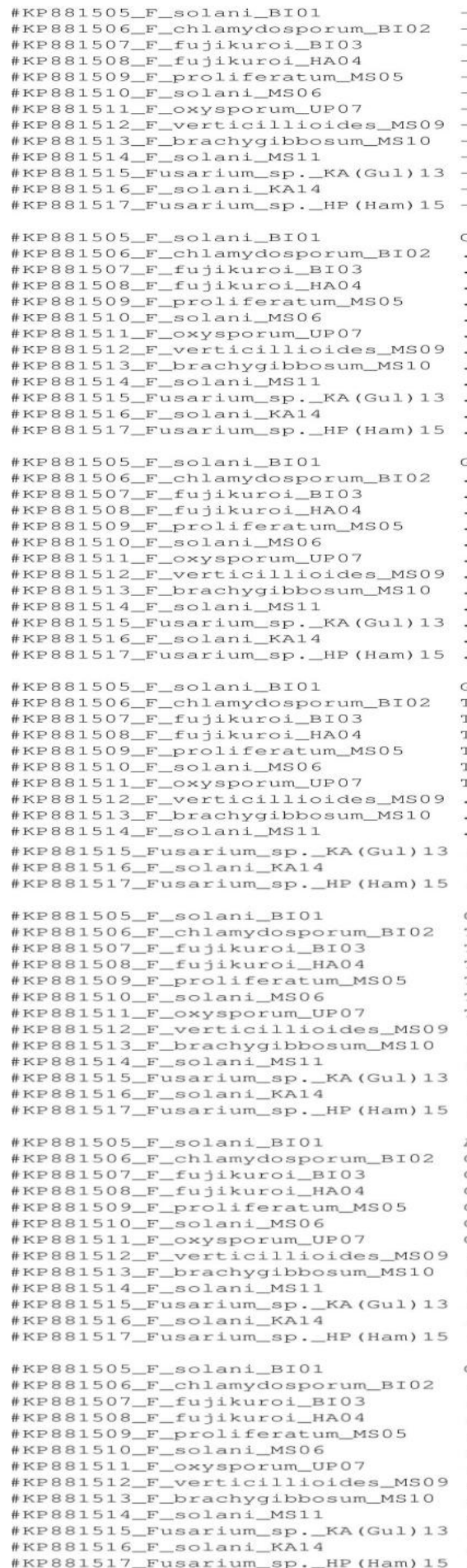

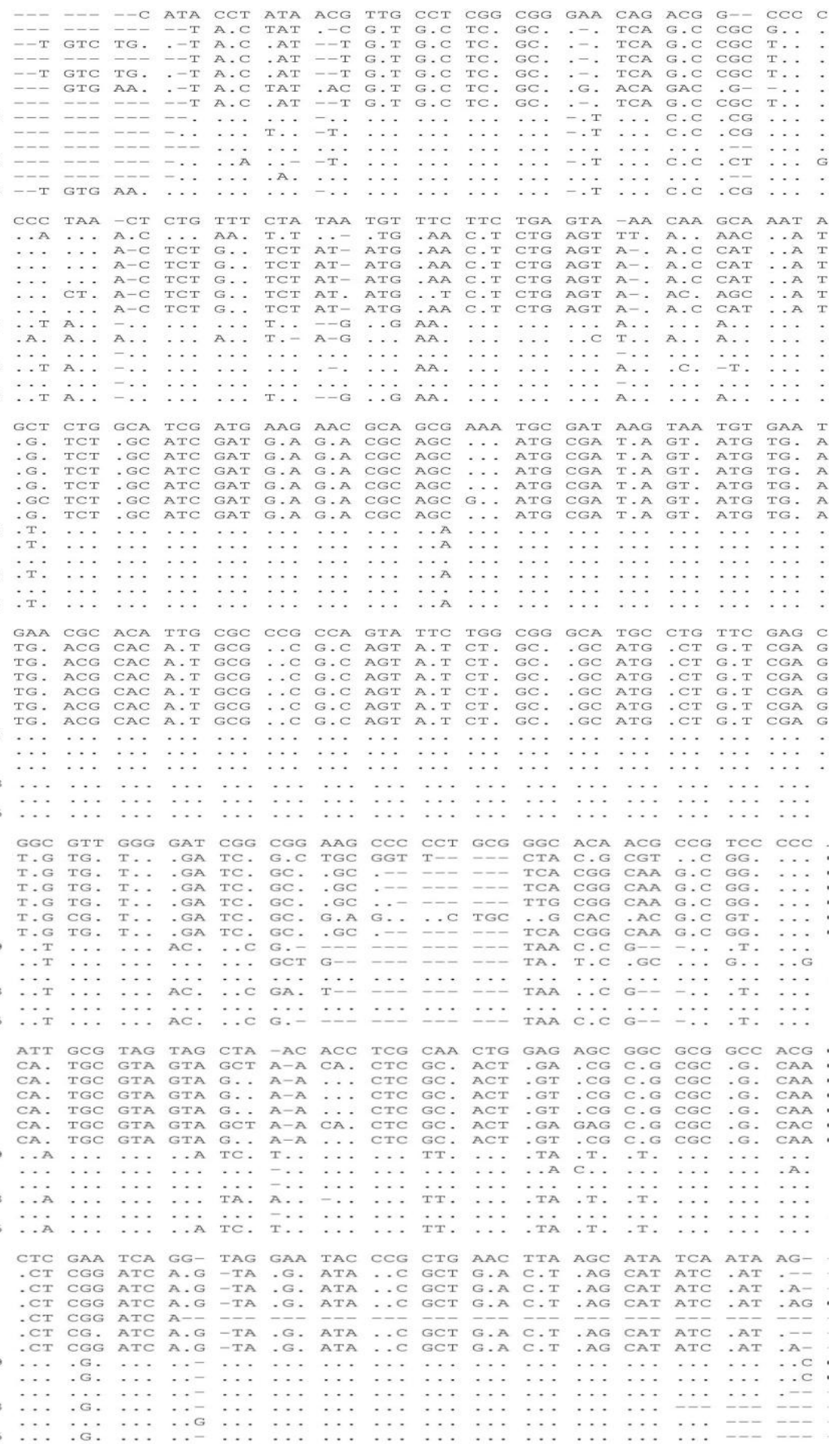


Fig.3 Typical microscopic photos of microconidia, macroconidia and chlamydospores of Fusarium isolates, scale bar length-20 $\mu \mathrm{m}$

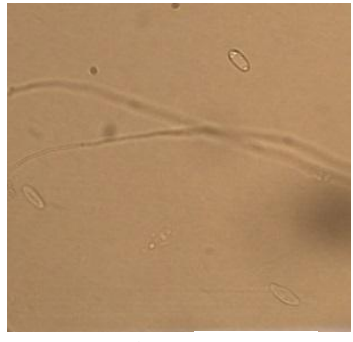

F. solani (BI01)

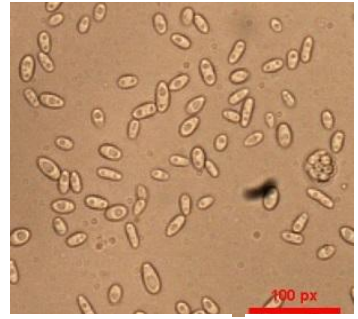

F. tabacinum(MS05)

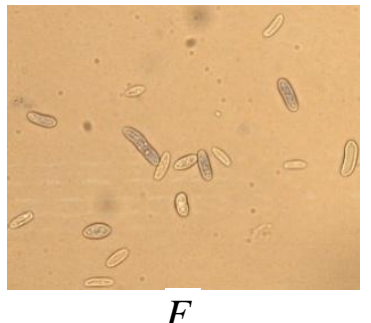

brachygibbosum(MS10)

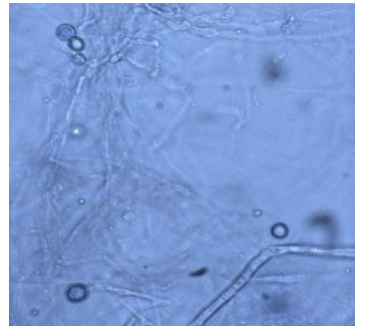

F. chlamydosporum (BI02)

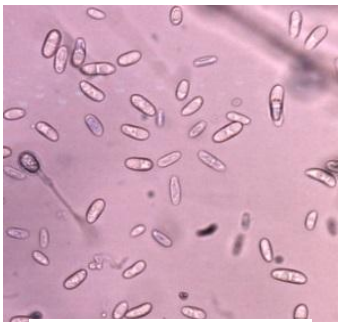

F. solani(MS06)

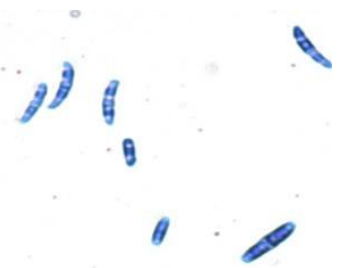

F. solani(MS11)

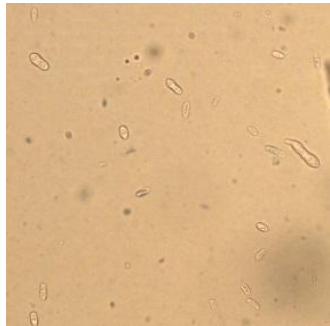

F. fujikuroi (BI03)

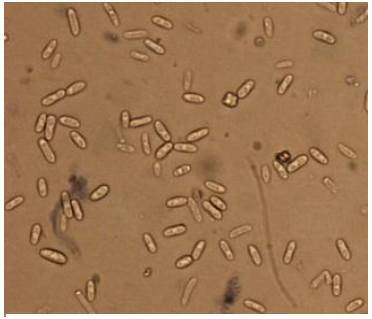

F. oxysporum(UP07)

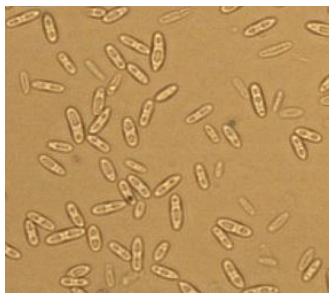

Fusarium sp.(KA(Gul)13)

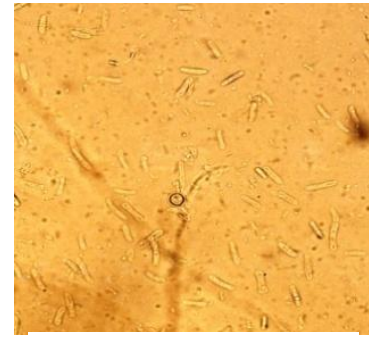

F. fujikuroi(HA04)

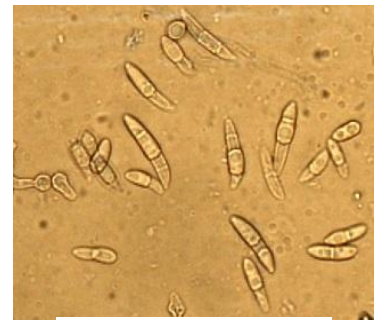

F. verticillioides

(MS09)

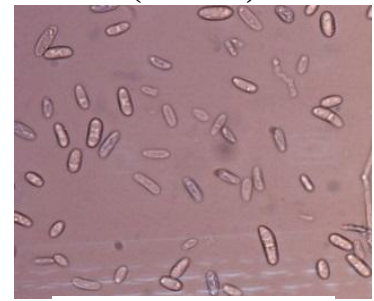

F. solani(KA14)

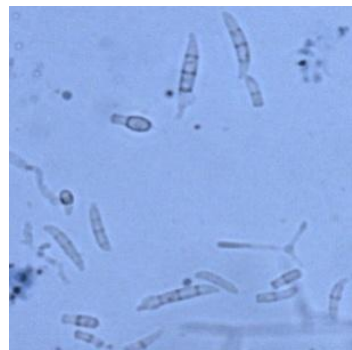

\section{F. incarnatum (HP(Ham)15)}

Phylogenetic analysis of the strains based on maximum likelihood method resulted into two major clusters which are further divided into two subclusters each. Subcluster I of Cluster I formed with three $F$. solani and subcluster II of cluster I formed with two Fusarium sp. and one $F$. verticillioides. Subcluster I of cluster II formed with one $F$. solani and subcluster II of cluster II formed with one $F$. proliferatum, two $F$. fujikuroi and one $F$. oxysporum. $F$. brachygibbosum and $F$. chlamydosporum were separated from both clusters (Figure 1). ITS sequences of all Fusarium isolates were aligned with the consensus region using CLUSTAL W program. The sequences are padded with gaps (dashes) so that wherever possible, columns contain identical characters from the sequences involved. Local alignment 
methods find related regions within sequences, they can consist of a subset of the characters within each sequence.

Sequence alignment is used to study the evolution of the sequences from a common ancestor such as protein sequences or DNA sequences. Mismatches in the alignment correspond to mutations, and gaps correspond to insertions or deletions (Figure 2).

In the present study, all 13 isolates of Fusarium spp. were morphologically identified and species determination was confirmed by PCR with primers specific for the ITS-rDNA region. Nucleotide sequences were later deposited with the NCBI GenBank database. The results showed that, out of the 13 isolates, four were $F$. solani, two were $\mathrm{F}$. fujikuroi, two were Fusarium sp., remaining each of one isolate was $F$. chlamydosporum, $F$. proliferatum, $F$. verticillioides, $F$. oxysporum and $F$. brachygibbosum are challenging to identify and to establish systems of taxonomy, because of strains were morphologically similar.

All Fusarium sp. were plant pathogens (Chandra et al., 2009). Isolate MS05 was identified as $F$. tabacinum morphologically but it was identified as $F$. proliferatum molecularly. To overcome these limitations of classical taxonomic and morphological characters for species discrimination of the genus Fusarium, molecular approaches to differentiate taxa is emphasized. Molecular tools like ITS provide necessary information required for a taxonomic purpose for species identification, as well as to elucidate the evolutionary relationships among species (Singha et al., 2016) and are reliable (Nadia et al., 2021).

\section{Acknowledgements}

The authors acknowledge the support of
ICAR-National Centre for Integrated Pest Management, Pusa Campus, New Delhi where all the research work has been conducted.

\section{References}

Baayen, R. P., K. O’Donnell, P. J. M. Bonants, E. Cigelnik, L. P. N. M. Kroon, E. J. A. 2000. Roebrock and Walwijck, C. Phytopathology. 90: 891907.

Bacon, C. W. and Hinton, D. M. 1996. Symptomless endophytic colonization of maize by Fusarium moniliforme. Can. J. Bot. 74: 1195-1202.

Bashyal, B. M., K. Rawat, S. Sharma, D. Kulshrestha, A. K. Singh, H. Dubey, A. K. U. Solanki, T. R. Sharma and Aggarwal, R. 2017. Whole genome sequencing of Fusarium fujikuroi provides insight into the role of secretory proteins and cell wall degrading enzymes in causing bakanae disease of rice. Frontiers in Plant Science.8: $1-12$. https://doi.org/10.3389/fpls.2017.0201 3.

Booth, C. 1971. The Genus Fusarium, Common wealth Mycological Institute, Kew.

Bottalico, A. and Perrone, G. 2002. Toxigenic Fusarium species and mycotoxins associated with head blight in smallgrain cereals in Europe. European Journal of Plant Pathology. 108: 611624.

Chandra, N. S., S. A. C. Udaya, S.R. Niranjana, M.S. Reddy, H. S. Prakash and Mortensen, C. N. 2009.Control of Fusarium verticillioides causing ear rot of maize by Pseudomonas fluorescens. PestManag Sci.65: 769-775.

Fracchia, S., I. Garcia-Romera, A. Godeas and Ocampo, J. A. 2000.Effect of the saprophytic fungus Fusarium oxysporum on Arbuscular mycorrhizal 
colonization and growth of plants in greenhouse and field trials. Plant Soil. 223: $175-184$.

Gerlach, W. and Nirenberg, H. 1982. The genus Fusarium-A pictorial atlas. Mitteilungenaus der Biologischen Bundesanstalt Für Land- und Forstwirtschaft (Berlin - Dahlem). 209:1-405.

Gupta, A. K., I. S. Solanki, B. M. Bashyal, Y. Singh and Srivastava, K. 2015. Bakanae of rice-an emerging disease in Asia. Journal of Animal and Plant Science. 25: 1499-1514.

ICRISAT. 1985. Annual report 1984. ICRISAT, Patancheru, 502 324, India.

Jalali, B. L. and Chand, H. 1991. Chickpea wilt. In: Diseases of International Importance, Vol. 1 (A. N. Mukhopadhya, H. S. Chaube, U. S. Singh, J. Kumar, ed.), PrenticeHall, New Jersey, USA.

Kheterpal, R. K. 2006.Ind. J. Phytopathol. 59: 397-398.

Kornerup, A. and Wancher, J. H. 1978. Methuen Handbook of Colour.3rd ed. Eyre Methuen Ltd, London.

Kumar, S., G. Stecher, M. Li, C. Knyaz and Tamura K. 2018. MEGA X: Molecular Evolutionary Genetics Analysis across computing platforms. Molecular Biology and Evolution. 35:1547-1549.

LeBlanc, N., A. Essarioui, L. Kinkel and Kistler, H. C. 2017. Phylogeny, plant species, and plant diversity influence carbon use phenotypes among Fusarium populations in the rhizosphere microbiome. Phytobiomes J. 1: 150-157.

Leslie, J. F. and Summerell, B. A. 2006. The Fusarium laboratory manual.1st ed. Blackwell Publishing Ltd, Oxford, London.

Martyn, R. D. 2014. Fusarium wilt of watermelon: 120 years of research. In Horticultural Reviews: Volume 42;
John Wiley \& Sons, Inc. Hoboken, NJ, USA., pp. 349-442.

Mohd Zainudin, N. A. I., A. A. Razak and Salleh, B. 2008.Bakanae disease of rice in Malaysia and Indonesia: etiology of the causal agent based on morphological, physiological and pathogenicity characteristics. Journal of Plant Protection Research. 48(4): 475-485.

Nadia, A., E. Stefańczyk, S. Sobkowiak, S. Chihat, H. Boureghda and Śliwka, J. 2021. Identification and pathogenicity of Fusarium spp. associated with tuber dry rot and wilt of potato in Algeria. Eur J Plant Pathol. 159: 495-509.

O'Donnell, K., H. C. Kistler, B. K. Tachke and Casper, H. H. 2000. Proc. Natl. Acad. Sci. 97: 7905-7910.

Raeder, U. and Broda, P. 1985. Rapid preparation of DNA from filamentous fungi. Letters in Applied Microbiology.1:17-20.

Rangaswami, G. 1958. An agar blocks techniques for isolating soil microorganism with special reference to pythiacious fungi. Science and Culture.24:85, 1958.

Rocha, O., K. Ansari and Doohan, F. M. 2005. Effects of trichothecenemycotoxins on eukaryotic cells: a review. Food Additives and Contamination. 22: 369378.

Singha, I. M., Y. Kakoty, B. G. Unni, J. Das and Kalita, M. C. 2016. Identification and characterization of Fusarium sp. using ITS and RAPD causing fusarium wilt of tomato isolated from Assam, North East. India Journal of Genetic Engineering and Biotechnology. 14: 99-105.

Skovgaard, K., H. I. Nirenberg, K. O’Donnell and Rosendahl, S. 2001. Phytopathology. 91: 1231-1237.

Summerell, B. A. B.Salleh and Leslie, J. F. 2003. A Utilitarian Approach to 
Fusarium Identification. Plant Disease. 87(2): 117-128.

Sunani, S. B. M. Bashyal, K. Rawat, C. Manjunatha, S. Sharma, G. Prakash, S. G. Krishnan, A. K. Singh and Aggarwal, R. 2019. Development of PCR and loop mediated isothermal amplification assay for the detection of bakanae pathogen Fusarium fujikuroi. Eur J Plant Pathol. 154:715-725.

Tamura, K. and Nei, M. 1993. Estimation of the number of nucleotide substitutions in the control region of mitochondrial DNA in humans and chimpanzees. Molecular Biology and Evolution. 10: $512-526$.

Vincelli, P. and Tisserat, N. 2008. Plant Dis. 92: 660-669.

White, T. J., T. Bruns, S. Lee and Taylor, J. 1990. Amplification and direct sequencing of fungal ribosomal RNA genes for phylogenetics. In: PCR Protocols: a Guide to Methods and Applications (M. Innis, D. Gelfand, J. Sninsky and T. White, eds.), Academic Press, Orlando, Florida, Chapter 38, pp.315-322.

Xiong, W. and Zhan, A. 2018.Testing clustering strategies for metabarcoding-based investigation of community-environment interactions. Mol. Ecol. Resour. 18: 1326-1338.

Zhang, Y. and Ma, L. J. 2017.Deciphering pathogenicity of Fusarium oxysporum from a phylogenomics Perspective. Adv. Genet. 100: 179-209.

Zhou, X. G., K. L. Everts and Bruton, B. D. 2010. Race 3, a new and highly virulent race of Fusarium oxysporum f. sp. niveum causing Fusarium wilt in watermelon. Plant Dis. 94: 92-98.

\section{How to cite this article:}

Monika Singh, O. P. Sharma and Someshwar Bhagat. 2021. Phenotypic and Molecular Identification of Pathogenic Fusarium Species Isolated from Various Pulse Growing Geographic Areas of India. Int.J.Curr.Microbiol.App.Sci. 10(11): 120-132.

doi: https://doi.org/10.20546/ijcmas.2021.1011.016 